\title{
Assessment of Children with Autism Spectrum Disorder and Post- Assessment Meeting with Parents: Some Issues of Planning and Professional Attitude
}

\author{
D. Terziev ${ }^{1,2, *}$, H. Manolova ${ }^{1,2}$, S. Staykova ${ }^{1,2}$ and M. Hristova ${ }^{1,2}$ \\ ${ }^{1}$ Clinic of Child Psychiatry "St. Nikolas", University Hospital Alexandrovska, Sofia, Bulgaria \\ ${ }^{2}$ Medical University of Sofia, Dpt. of Psychiatry and Medical Psychology, Bulgaria
}

\begin{abstract}
The authors, child psychiatrists and clinical psychologists, conducting team assessment of children with developmental disabilities (including autism spectrum disorder) for more than two decades, present some important in their view issues and recommendations concerning planning and proceeding of assessment and post-assessment meetings with parents. It is essential to ensure not only an accurate assessment protocol and diagnosis, but also to "attune" to parents in order to facilitate their understanding and more positive use of assessment outcome results. Parents need further support to "survive" psychically the post - assessment period and to provide for adequate interventions for their child.
\end{abstract}

Keywords: ASD, children, parents, assessment, post-assessment meeting, assessment team.

The diagnostic assessment and facing its outcomes is a crucial time for parents of children with Autism Spectrum Disorder (ASD). As an emotional experience depending on people and families with their historical and current backgrounds it triggers a mixture of relief after a long waiting, post-assessment shock and urge for mobilization, and frequently self-reproaches and anxiety in view of immediate and remote future.

Along with screening and interventions, assessment is a part of the organized professional care for children with ASD and their families [1]. It helps parents to understand and put into diagnostic frame child's deficits and aberrant behavior, gives them a "diagnostic pass" to the systems of supports. Thus parents can enter the "common rails" and feel less alone. However, assessment results might put them in the traps of denial, anger (toward professionals, others, self, destiny) and depression; it might aggravate their internal and interpersonal conflicts and even lead to separation and abdication of one of them - in short it is not so easy for parents to survive psychically the assessment process.

Mother of an autistic child, diagnosed at 3years, shares her feelings at that time "I was so lonely and desperate. At the same time I was angry with myself and my husband, who was keeping denying the problem and didn't wanted to do anything and I was in line with him I hated you most, because you told me the diagnosis!"

*Address correspondence to this author at the Clinic of Child Psychiatry "St. Nikolas", University Hospital "Alexandrovska", 1, G. Sofiiski Blvd., Sofia 1431, Bulgaria; Tel: +35929230375; E-mail: dislter@yahoo.co.uk
The assessment and resulting diagnosis are important mark in the parental turmoil and journey of raising a child with mental disability, a journey that can even spur families to examine their belief systems [2]. Starting with a burdensome experience, however, some parents succeed to make positive adaptations in the form of changed worldviews concerning life and disability, and with recognition of the positive contributions made by their child to themselves [3].

The whole atmosphere and not only the activities of assessment, the way it is planned and data communicated to parents and discussed with them, undoubtedly interfere with the achievements of the above mentioned positive effects of it. Professionals' stance of attunement to parents and their possible inner experiences may facilitate the positive outcome of assessment process. Attunement as a term is due to Daniel Stern describing chain affects exchange between mother and bigger baby [4]. Here by attunement we mean a professional attitude of intentness to understand experiences and points of parents and convey empathy in the frame of viewing contact with them as intersubjective experience and not as one-sided intervention.

What follows is an attempt to summarize and briefly discuss some issues to be kept in mind when assessment is planned, conducted and its outcomes reported to parents.

\section{ISSUES AND RECOMMENDATIONS CONCERNING ASSESSMENT}

In the desirable case the followings are met: 
1. Parents are prepared for the assessment process by preliminary consultations with the team member. Procedure, assessing professionals, diagnostic instruments, parental responsibilities, the extent of their participation and other aspects are clearly stated and discussed.

"Before going to the clinic I was afraid that everything would be scary and all of us would be assessed. Even me and my husband. After the first consultation I calmed down quite a lot. They explained to me what represent the assessment itself, who was going to make it and how long it would take. I understood the aim would not be just the diagnosis, but assessment of the progress, strengths, not only difficulties of my child."

2. Both parents (or one parent if single and a significant other) are invited to take part in the assessment. Thus they may support and complement each other, coordinate their views and understandings of the child and raise more topics. Child's relating to both adults may be observed together with the ease of each one of them to engage the child in interaction,

"I heard for the first time my husband sharing his impressions on our son. He said things I would not consider important but turned out of a great importance for the assessment...He was always avoiding the issue, thinking even useless going there"

3. Assessment is done thoroughly and professionals are strict and precise in their professional roles and behaviours. This makes it easier for parents to hear conclusions and start "metabolizing" them.

4. Assessment is providing opportunity for both parents to be present directly in the diagnostic sessions or to observe them on distant monitors. Videotaped sessions may be discussed within the assessment team or be viewed jointly with parents and discussed, which makes videotapes especially valuable. Putting the parents in the roles of observers, participants and discussants, may create some difficulties but will assure a common and concrete "material" to be used when presenting assessment results.
Parents of a 3-yars old child with ASD: "The fact they allow us to attend the assessment was very helpful. We were distressed by the fact it could not do everything, but we saw how it could help him to succeed."

\section{ISSUES AND RECOMMENDATIONS CONCERNING POST-ASSESSMENT MEETING}

In view of so-called "feedback session" or a "family conference" [5] we find the followings important :

1. Before the meeting with parents a thorough professional team discussion facilitates formulation of a common conclusion. Conflicting professional opinions in terms of content or reflecting interpersonal tensions may be an obstacle.

2. In the best scenario, all professionals of the assessment team take part of the meeting. One of them in the role of principal spokesman is responsible to direct and to some extent structure the meeting.

3. If the final assessment conclusions and recommendation are completed after meeting they may be composed in a way more coinciding with its content and thus more recognizable by parents.

Mother of a 4-years old child with ASD: "When I read the assessment report after the final session, I got far more easily what was written. I saw in it my child indeed, not just terms, saying nothing to me."

4. At the meeting parents are experientially and emotionally where they are, so trying to tune to them is to start from their point. The questions like "How did you (and each of you separately) feel during the assessment?" "What was the most difficult?" "What is the most important for you to start this discussion with?" may be useful. Such an opening invites for a dialogue and more active parental participation.

Parents of a 5 - years old: "When we meet specialists, they say something and we just listen. Seldom they are interested in what we think, what we need and how we feel as parents...." 
5. Not only questions asked and the statements made by parents but also topics expected but not raised by them are important. The most emotionally charged questions may be suspended or asked at the door while leaving. Such "at the door question" may be for example: "Will he/she be like the others one day?" Such questions should be dealt with seriousness and in the best case discussed in an additional session.

6. Information in terms of content (what we say to parents) has three main and logically connected topics: what are the problems with the child? What to expect? What can be done? However, the after-assessment meeting, hopefully, proceeds as an interaction and conversation with parents and not as a speech. The parents cannot be expected to hear, understand and keep in mind everything. They need some "digestion time" after receiving feedback session information and this could be facilitated if an additional meeting is proposed.

Mother of a 4 -years old child with ASD: "At the final meeting me and my husband got different things. I hear one thing, he something else. The next meeting helped us to understand results better."

7. Both deficits and strengths of the child should be pointed out and explained to the parents, leaning on concrete and shared with them observations whenever possible.

Parents of a 6 -years old child with ASD: "It was very helpful to hear not only what is not possible, but what is possible and what is good to focus on."

8. The categorical diagnosis and the way of using and grouping data in order to make it should be explained to parents. They may need further explanations in view of the plethora of diagnostic terms related with ASD.

9. What the parents need is a (realistic) hope and it is to be facilitated. Without any hope they would be depressed and incapacitated.

Father of a 5-years old child with ASD: "When I heard the diagnosis for the first time, I was completely desperate. Doing something didn't make sense to me, the existence of the child, of us didn't make sense to me at all! All I wanted was to hear whether he can become a person at all!"

Sensing the boundary between what could be a realistic hope for a particular family in a particular situation and what is their capacity to hear the child's problems at the moment is of most importance.

10. It is helpful to think not only of the communicative content of the meeting but also of the communicative and interactional processes. This might be facilitated by process-interruption questions [6] or statements. For example: "What do you suppose yours comments of this may be at home?" Empathic statements may be used at appropriate times during the meeting. Here, the nonverbal accompaniment of the statements is of prime importance.

11. In some cases parents may remain silent and even silent for a long period. Questions such as "What do you think about this?" "Do you think this is correct?" may be used but the pressure is to be avoided. We may comment on the silence but the intention behind such comments matters the most - in the good case it will be not an attempt to break the silence of the parents but rather to understand and support their emotional experiences.

12. What parents say or ask should be listened to, heard, understood and only then answered. Listening skills are the most important in the communicative process. Listening may be at times active or reflexive, that is an active search to understand better what parent(s) are saying or intended to say. Statements and questions like "If I understand you correctly you ......." or "What do you mean by.......?" may be of help.

13. Talking more does not mean saying more and even the reverse is frequently true. Saying a lot does not help parents to hear and understand.

Mother of a 3-years old child: "They were telling us so many and so difficult things, as if we were drowning into information. I wanted them to stop."

In order to be successful, every session should have some focus and some issues repeated and rephrased. Professionals must be able not only to talk 
but also to accept, feel comfortable with, and hold some periods of not talking.

14. Professionals must be ready to deal with rejection of the opinions they give to parents, rationalizations, denigration of the assessment process. They must be attentive to triggers of these behaviours, such as real shortcomings of the assessment procedures as well as emotional defensive parental reactions. The professional attitude here is of prime importance and preserving the attitude of support and empathy in the "hot" moments of the meeting is crucial. Here professionals need both professional competence and personal maturity.

15. We often talk of parents as a whole unit but in fact they are different. They may have and certainly have their more or less conflicting and more or less openly conflicting relationships. The problems with the child and what has happened during the assessment and the meetings after it, interferes with their relationships. Their behaviours as every human's behaviour are overdetermined and reflective of their relationship. At the other hand, their capability to work as a team and their capacity to remain organized probably after some period of turmoil and instability are of vital importance. One of the parents may remain silent during the meeting leaving the space for the other to speak. Nonverbal messages of the parents may be different and/or may differ from the verbal content. We must note that difference but to great extent respect it. If sensing it as appropriate the silent parent may be asked directly about his opinion or indirectly through the other parent: "If I ask him what is his opinion on this, and if he is ready to answer, what do you suppose he will say?" or "What, do you think, his comment on this could be after you leave?" Then the correctness of the answer may be checked with the silent parent. In any case, supporting both parents is obligatory and we must intervene sensitively respecting the moment, the content and the process in any time of the meeting.
16. In after-assessment meeting with parents, as in another kind of sessions lead by a helping professionals, the responsibility is to "hold" the situation. In our case, this will imply to be in sensitive care about achieving the aims of the meeting as much as possible. This implies taking care of parents, the physical conditions of the meeting like space time and boundaries, the content and the process of interaction. It is obvious that professionals actively involved in and responsible for after-assessment meetings may significantly profit if having some interrelational and systemic professional competences and expertise, apart from their knowledge and skills related to ASD. From interrelational point of view, the awareness of social and cultural stereotyping, transference, counter transference and the openness to our own contribution to the quality of interactions are important. Both families and the institution we are working in, are systems (evolving in an evolving context) and appraisal of their functioning and areas of dysfunction in terms of subsystems, of hierarchy and of communication are to be considered.

\section{REFERENCES}

[1] Filipek PA, et al. Practice parameter: Screening and diagnosis of autism. Report of the quality standards subcommittee of the American Academy of Neurology and Child Neurology Society Neurology 2000; 55: 468-79.

[2] King G, Zwaigenbowm L, King S, Baxter D, Rosenbaum P. A qualitative investigation of changes in belief systems of families of children with autism or Dawn syndrome. Child: Care, Health \& Development 2006; 32; 353-369.

https://doi.org/10.1111/j.1365-2214.2006.00571.x

[3] Woodgate RL, Ateah C, Secco C. Living in a world of their own The experience of parents who have a child with autism. Qualitative Health Research 2008; 18; 1075-1083. https://doi.org/10.1177/1049732308320112

[4] Stern DN. The Sense of a Subjective Self: II. Affect Attunement. In Stern DN. The Interpersonal World of the Infant. A View from Psychoanalysis and Developmental Psychology. London: Karnac Books 1998; pp. 138-161.

[5] Bailey K. Supporting families. In: Katarzyna Ch, Klin A, Volkmar FR, editors. Autism spectrum disorders in infants and toddlers. Diagnosis, assessment and treatment. New York, London: Guilford Press 2008; pp. 300-26.

[6] Tomm K. Interventive interviewing: Part II: Reflexive questioning as a means to enable self - healing. Fam Proc 1987; 26; 167-83. https://doi.org/10.1111/j.1545-5300.1987.00167.x

(C) 2017 Terziev et al.; Licensee Lifescience Global.

This is an open access article licensed under the terms of the Creative Commons Attribution Non-Commercial License (http://creativecommons.org/licenses/by-nc/3.0/) which permits unrestricted, non-commercial use, distribution and reproduction in any medium, provided the work is properly cited. 